\title{
AN EPOXY-STAMP ON GLASS-DISC SPECIMEN EXHIBITING STABLE DEBONDING FOR IDENTIFYING ADHESIVE PROPERTIES
}

\author{
A. Sekulic, A. Curnier \\ Laboratoire de mécanique appliquée et d'analyse de fiabilité (LMAF) \\ Ecole Polytechnique Fédérale de Lausanne (EPFL) \\ CH-1015, Switzerland
}

\begin{abstract}
In Contact Mechanics and Tribology, adhesion is the conservative reversible normal relation (contiguity) and attractive interaction (tension) at the interface between two materials in contact. "Deadhesion" or rather decohesion, is the loss of adhesion. In Fracture Mechanics and Rheology, debonding is usually interpreted as a crack propagating along the material pair interface at the microscale and as damage at the macroscale.

This study is motivated by the fibre-matrix debonding which occurs in glass fibre reinforced epoxy matrix composites and decreases their strength. In this note, we focus our attention on the identification of the adhesive properties between glass and epoxy.

The basic adhesive property, entering a normal adhesion-decohesion law relating the normal tensile stress $p_{n}$ to the normal gap $g_{n}$ at an interface, is the surface energy of adhesion (per unit area) $\omega$ (of Dupré). It represents the local work dissipated during the decohesion process (i.e. at the particle pair or continuum elementary area microscale). In several contact mechanics models of adhesion-decohesion, the surface energy $\omega$ is equal to the product of two other basic adhesive properties, namely the adhesion peak stress $\pi_{n}$ and the decohesion rupture gap $\gamma_{n}$, or rather a fraction of it (e.g. one half),

$$
\omega=\frac{1}{2} \pi_{n} \gamma_{n}
$$

These adhesive properties are difficult to measure accurately because the decohesion process is often unstable.

In this article, we present an original axisymmetric specimen composed of an epoxy-stamp bonded on a glass-disc, which exhibits a stable decohesion under traction and hereby permits an accurate identification of the adhesive properties. Debonding stability is obtained by combining two stabilizing effects of the epoxy stamp geometry : axisymmetry and concavity, discovered by analogy with two observations in contact and fracture mechanics, respectively. In case of axisymmetry, the crack area grows with the square of its radius $a^{2}$ (instead of its length $a$ in a plane strain geometry). Concavity is inspired from the Tapered Double Cantilever Beam (TDCB) used in experimental Fracture Mechanics [Kinloch and Young (1983) [1]. Optimization of the shape of the epoxy part was done by numerical simulations. To this end the Talon-Curnier contact-adhesion law [2] was used. According to that law, debonding is stable if the tension stress at the crack front decreases as the crack propagates, i.e. if the envelope of the tension stress at the crack front decreases along the radial axis. The simulations were carried out with the contact analysis program TACT. The final design of the axisymmetric concave epoxy-stamp on glass-disc specimen is shown in cross-section in Figure 1a and in perspective in Figure 1b.
\end{abstract}

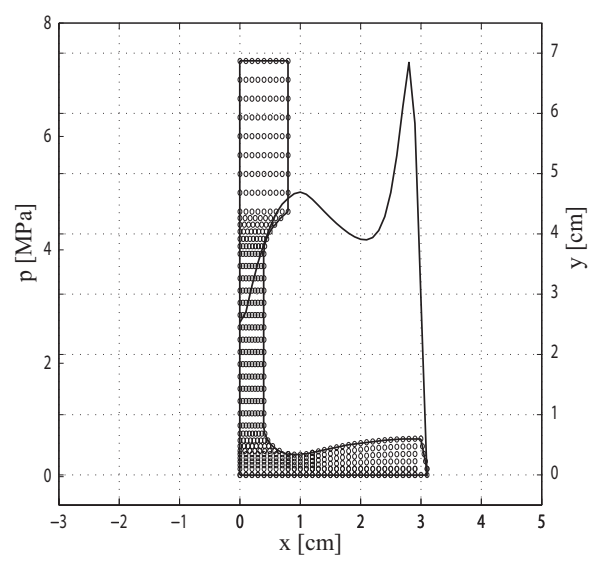

a) Cross-section with tension envelope

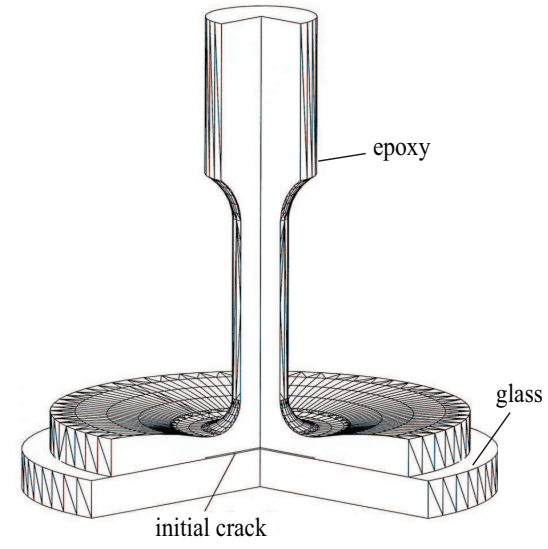

b) Perspective with cut sector

Figure 1: Axisymmetric concave epoxy-stamp on glass-disc specimen. 
The graph of the tension stress envelope at crack front superposed on the mesh in Figure 1a shows that the debonding process is stable over a large radial zone.

The specimen is then pulled on a standard traction machine (Instron 5800). A controlled displacement $w$ is imposed at the rate $\dot{w}=1 \mathrm{~mm} / \mathrm{min}$. The traction force $f$, the specimen elongation $u$ and the crack radius $a$ were measured.

The experimental results are presented in Figure 2. The force histograph $f=\mathrm{f}(t)$ is shown in Figure $2 \mathrm{a}$. The crack initiation (point $\mathrm{A}$ at radius $r$ ) and termination (point $\mathrm{B}$ at $R$ ) are easy to identify on this graph. It is clear that the debonding process is stable between A and B since an increasing force is necessary for advancing the crack from $r$ to $R$. The early discontinuity (point $\mathrm{C}$ ) corresponds to the (sudden) debonding of the mold release film interposed for initiating the crack.

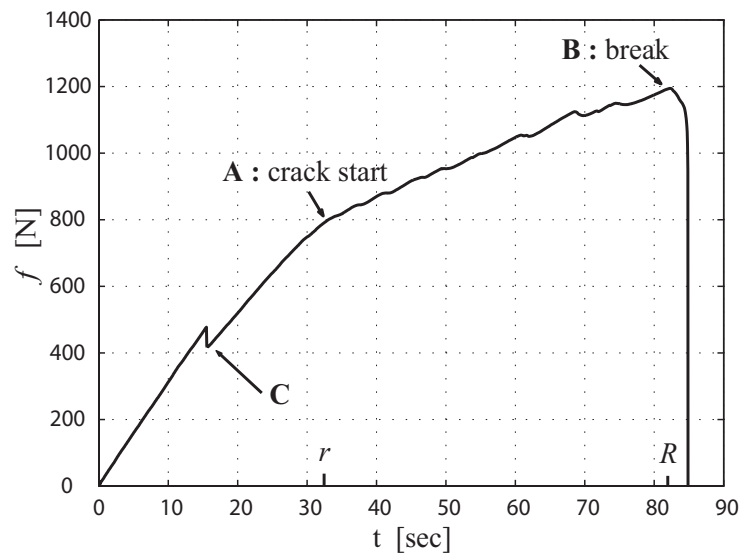

a) Force-time curve

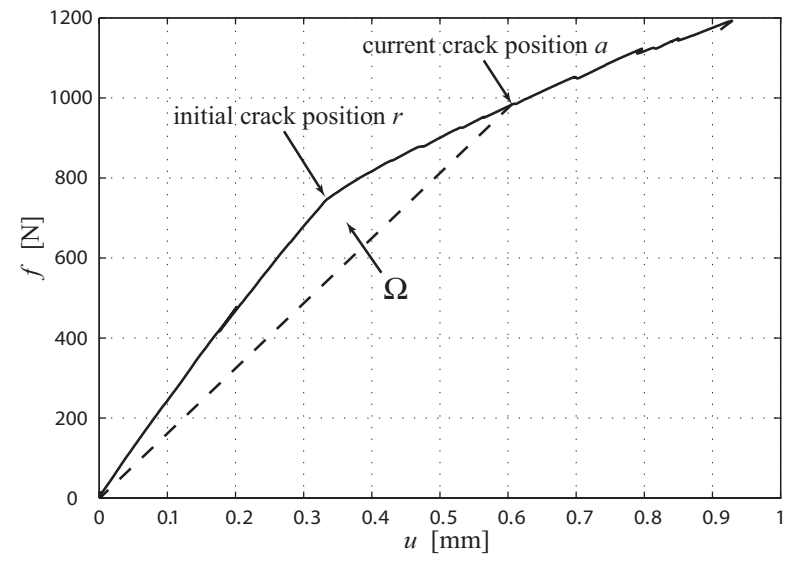

b) Force-displacement curve.

Figure 2: Force-time and force-displacement curve.

The adhesion properties (surface energy $\omega$, peak stress $\pi_{n}$ and rupture gap $\gamma_{n}$ ) can be extracted from the experimental measurements [Fig. 2b] (specimen elongation $u$, traction force $f$ and crack radius $a$ ) by the following procedure. The work dissipated between the initial crack radius $r$ and the current one $a$ is equal to the dissipation per unit area multiplied by the debonded area $A=\pi\left(a^{2}-r^{2}\right)$ :

$$
\Omega=\omega A=\frac{1}{2} \pi_{n} \gamma_{n} \pi\left(a^{2}-r^{2}\right)
$$

Firstly, this dissipated energy $\Omega$ is measured on the experimental force-displacement graph $f=\mathrm{f}(u)$ [Fig. 2b]. It is equal to the area of the hysteresis loop obtained upon elastic unloading. Elastic unloading can be either idealised as linear or measured from a real experimental unloading. Secondly, the measured traction force $f$ or prescribed specimen elongation $u$ at the initial crack radius $r$ is used as a boundary condition in a simulation of the stamp (free between 0 and $r$ and fixed between $r$ and $R$ at its basis), with the contact analysis program TACT, in order to evaluate the adhesion peak stress $\pi_{n}$.

Finally, the rupture gap $\gamma_{n}$ is calculated by solving equation (2) for it

$$
\gamma_{n}=2 \frac{\omega}{\pi_{n}}=2 \frac{\Omega}{\pi\left(a^{2}-r^{2}\right)} \frac{1}{\pi_{n}}
$$

The stamp on disc specimen design can be used for other pairs of materials (provided one can be molded on the other). Finally, it can perhaps be adapted into a torsion experiment to study adherence and decoherence. Combining torsion with compression on a torsion-traction machine may permit to find out whether adherence depends on pressure or not.

\section{REFERENCES}

[1] Kinloch, A., Young, J., "Fracture behaviour of Polymers", Applied science Publishers, 1983.

[2] Talon, C., Curnier, A., "A Model of Adhesion Coupled to Contact and Friction", European Journal of Mechanics A/Solids, Vol. 22, 545-565, 2003. 